\title{
Pycnandra acuminata, ein Endemit Neukaledoniens mit extremer Schwermetalltoleranz
}

\author{
Hubertus Nimsch \& Veit Martin Dörken
}

\begin{abstract}
Pycnandra acuminata is unquestionably one of the most conspicuous and interesting endemic plants of New Caledonia and is particularly remarkable for its tolerance to heavy metals occurring naturally in high concentrations in local soils there. Here we describe the pIant's ecological and biological features.
\end{abstract}

\section{Zusammenfassung}

Pycnandra acuminata gehört zweifelsohne zu den wohl bemerkenswertesten Endemiten Neukaledoniens mit einer ausgesprochenen Toleranz gegenüber Schwermetallstress. Ökologie und Biologie dieser Art werden nachfolgend vorgestellt.

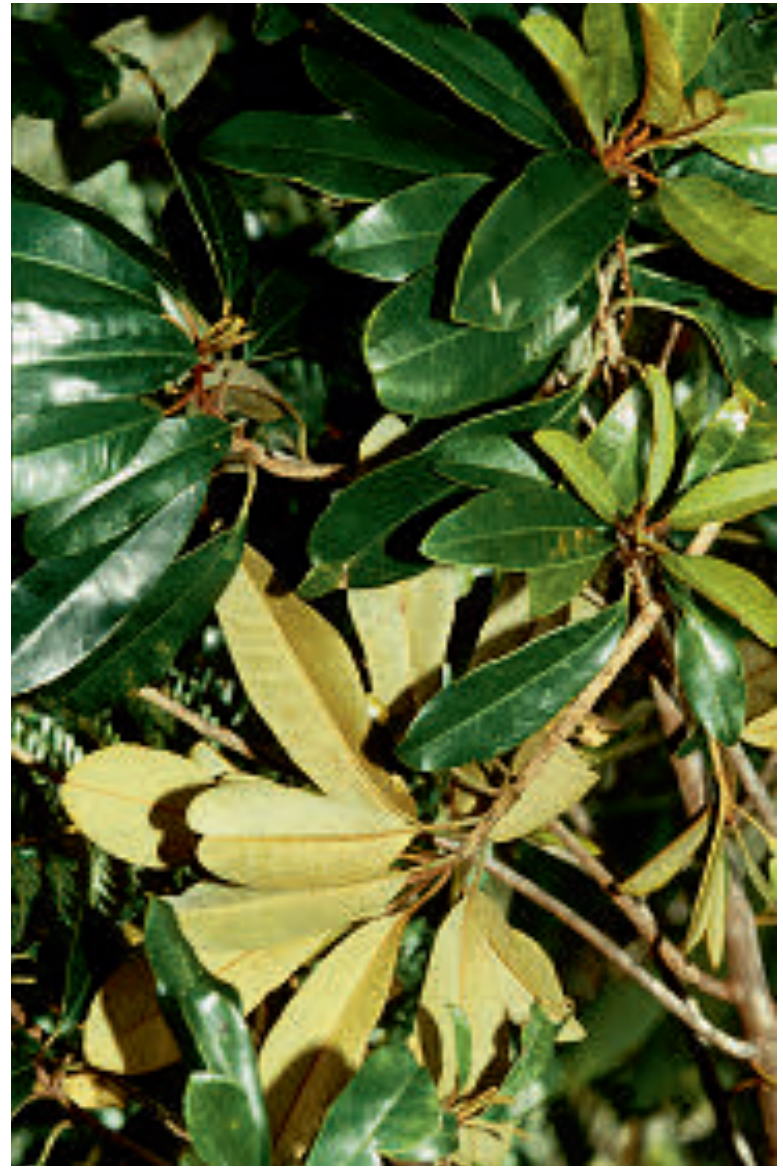

\section{Einleitung}

Die südpazifische Inselgruppe Neukaledonien ist sowohl geomorphologisch als auch klimatisch recht heterogen. Dies und eine rund 100 Millionen Jahre andauernde Isolation des Archipels führte zu einer extrem reichhaltigen Flora mit zahlreichen lokalen Endemiten (SChneckenburger 1991; Jaffré et al. 2001).
Einer der wohl bemerkenswertesten Endemiten Neukaledoniens ist Pycnandra acuminata (Baill.) Swenson \& Munzinger. Es handelt sich um ein habituell recht unauffälliges Gehölz, das jedoch über eine erstaunliche Physiologie verfügt, die es den Bäumen erlaubt, auch auf extrem schwermetallbelasteten Böden zu wachsen (JAFFré et al. 1976). Zwar gibt es auch in unserer heimischen Flora mit z. B. dem Gelben Galmei-Veilchen (Viola calaminaria) oder dem Violetten Galmei-Stiefmütterchen (Viola guestphalica) endemische Arten, die auf Schwermetallböden verbreitet sind, jedoch ist das Spektrum der bei uns in der heimischen Flora endemischen Schwermetallpflanzen im Vergleich zum Artenspektrum Neukaledoniens verschwindend gering. Neben Pycnandra acuminata sind auf Neukaledonien zahlreiche weitere Nickel-hyperakkumulierende Arten bekannt (Kersten et al. 1980; Callahan et al. 2012, JAFFré et al. 2013). Bislang sind sogar 65 Nickel-hyperakkumulierenden Arten (Lianen, Sträucher und Bäume) auf Neukaledonien nachgewiesen, was Neukaledonien zu einem der weltweiten Diversitätszentren für Schwermetallpflanzen macht (JAFFrÉ et al. 2013).

\section{Systematik}

Die Gattung Pycnandra ist auf Neukaledonien mit 12 Arten vertreten und gehört zur Familie der Sapotaceae (Breiapfelgewächse) aus der Ordnung der Ericales. Innerhalb der Sapotaceae wird die Gattung zur Unterfamilie der 


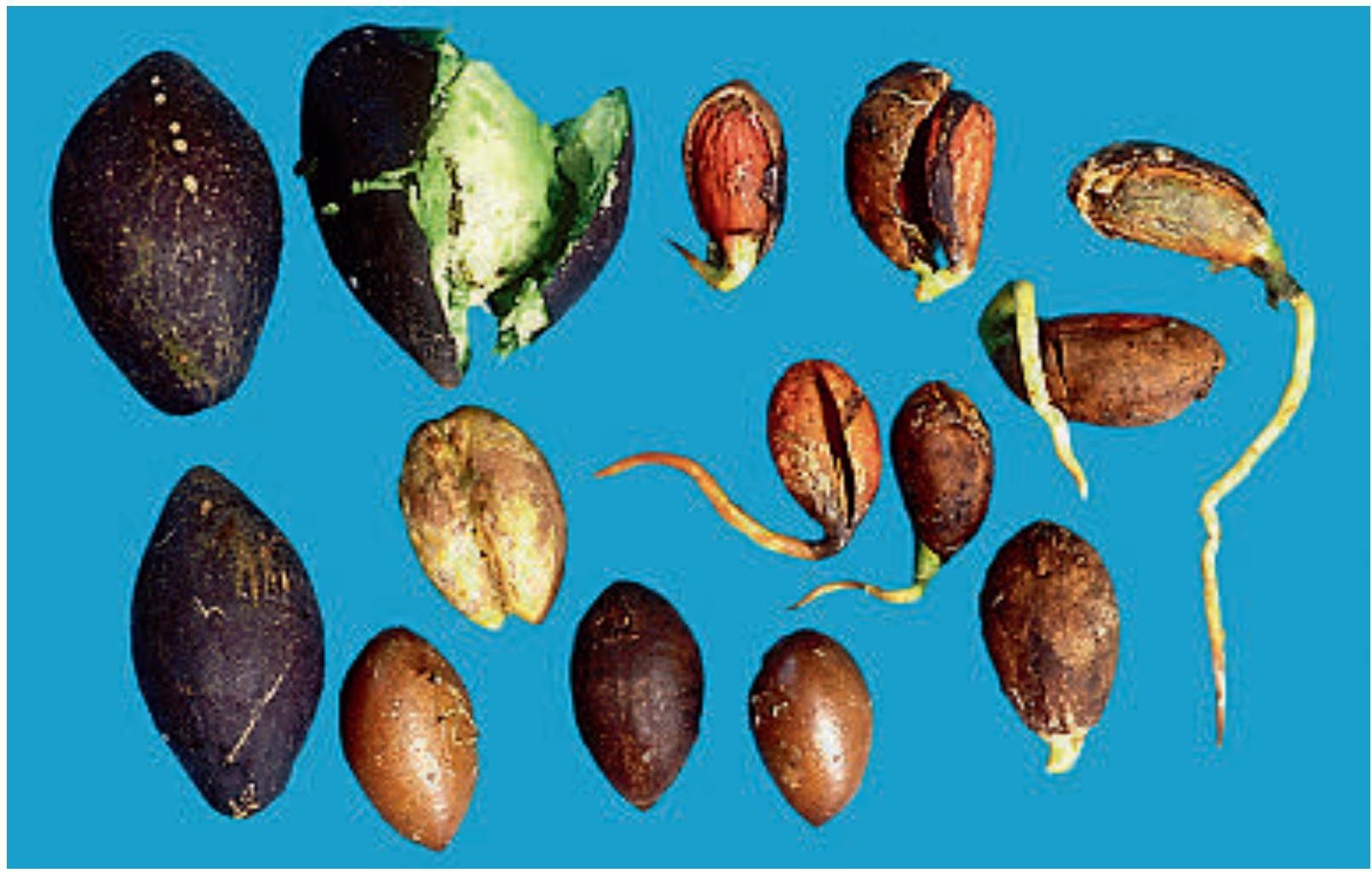

Chrysophylloideae gestellt. Für viele Arten der Flora Neukaledoniens wird die systematische Zuordnung immer noch kontrovers diskutiert, was einerseits in der stark eingeschränkten Verfügbarkeit des Pflanzenmaterials und anderseits auch in der Seltenheit der Arten im Gelände begründet ist. Daher unterliegen auch heutzutage zahlreiche neukaledonische Taxa immer wieder systematischen, taxonomischen und nomenklatorischen Änderungen. Dies spiegelt sich auch in der Zuordnung von Pycnandra acuminata wider. So wurde dieses Taxon in der Vergangenheit in sechs verschiedenen Gattungen geführt und als Chrysophyllum sarlinii Guillaumin, Niemeyera acuminata (Pierre ex Baill.) T. D. Penn. Pouteria acuminata (Pierre ex Baill.) Baehni, Sebertia acuminata (Pierre ex Baill.) Engl., Sersalisia acuminata Pierre ex BaIll. oder Trouettia sarlinii (GuILlaumin) AubrÉv bezeichnet.

Abb. 1 (Seite 120): Pycnandra acuminata, ausgewachsene Pflanze.

Abb. 2 (oben): Pycnandra acuminata, Früchte, Samen und Keimlinge.

\section{Verbreitung}

Pycnandra acuminata ist in den feuchten, niederschlagsreichen Regenwäldern Neukaledoniens heimisch. Die zweite auf Neukaledonien heimische Pycnandra-Art (P. gatopensis) ist hingegen nur ganz lokal im Süden der Grande Terre in Macchia-ähnlichen, sehr lockeren Pflanzenbeständen verbreitet. Sie wächst in der Nähe der Uferbereiche des Rivière des Lacs und des Creek Pernod - aber immer außerhalb der Überflutungsbereiche. Sie wurde auch auf dem Plateau de Yaté gefunden. Alle Standorte sind ultrabasisch und in hohem Maße schwermetallbelastet (JAFFré et al. 1976). Die Böden haben sich aus einem ultrabasischen Ausgangsgestein (Serpentin) entwickelt, welches für die hohen Anteile an Magnesium, Eisen, Kobalt Nickel und Kadmium verantwortlich ist (CALlahaN et al. 2012). Der hohe Eisenerzanteil (mit bis zu $75 \%)$ ist der Grund für die rotbraune Färbung dieser Böden.

\section{Der Strauch}

Pycnandra acuminata ist ein Großstrauch, der aber auch gelegentlich als kleiner, bis $13 \mathrm{~m}$ hoher Baum bei einem Stammdurchmesser bis 

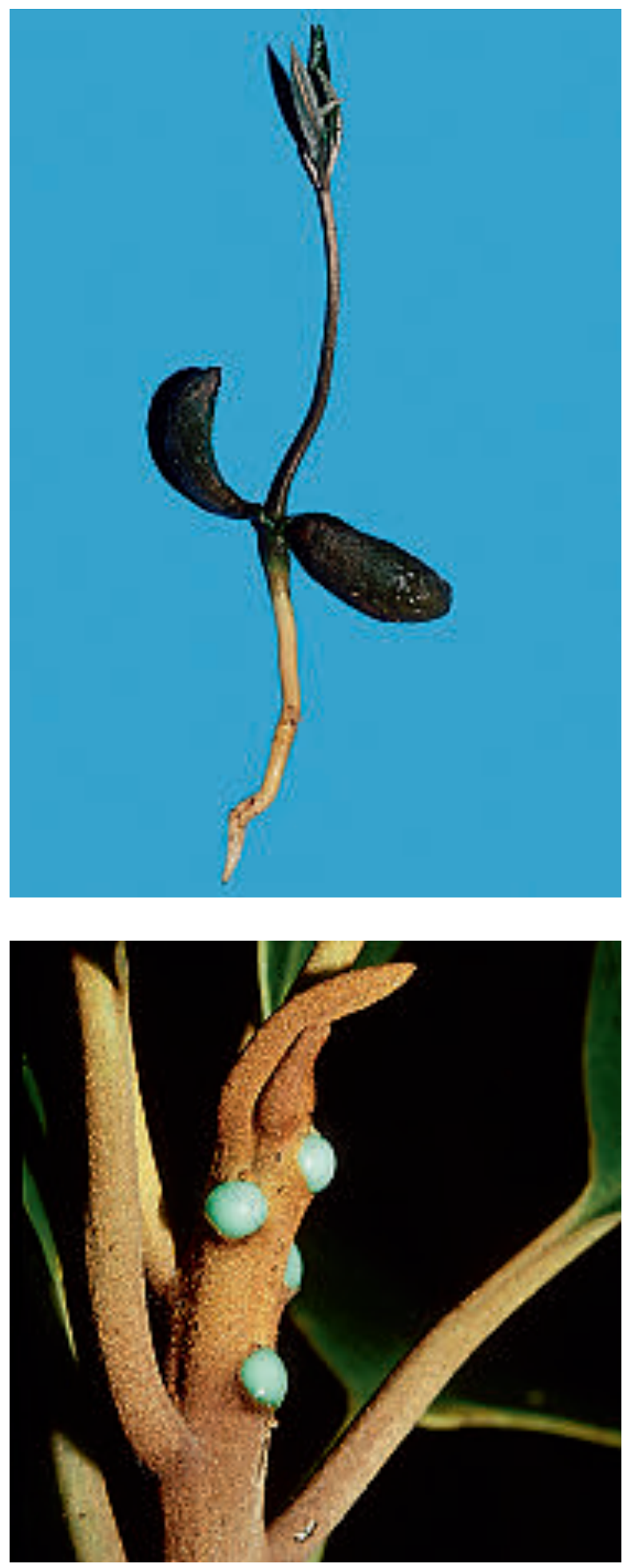

Abb. 3 (oben links): Pycnandra acuminata, Keimling mit ersten Blättern.

Abb. 4 (oben rechts): Pycnandra acuminata, Keimling.

Abb. 5 (unten rechts): Pycnandra gatopensis, erwachsene Pflanze mit Früchten, auf Macchia-ähnlichen, trockenen Standorten, Rivière des Lacs.

Abb. 6 (unten): Pycnandra acuminata, austretender Latexsaft, durch Nickelcitrat grünblau gefärbt.
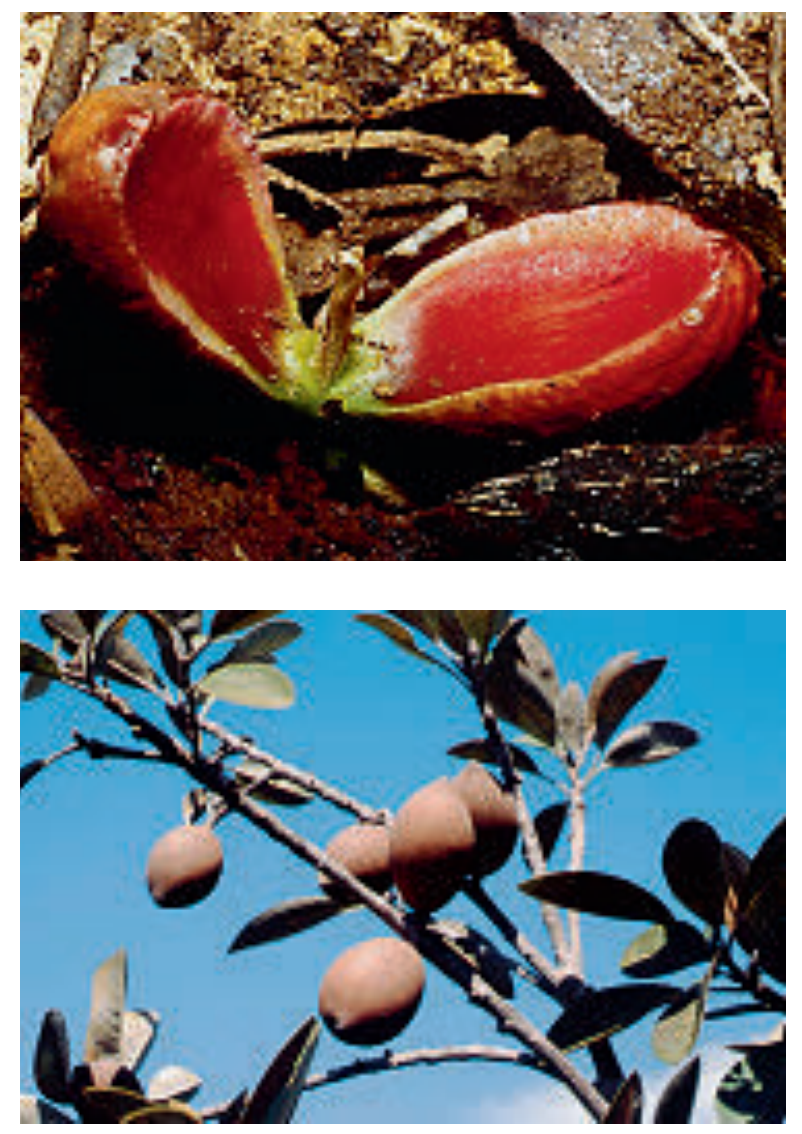

$70 \mathrm{~cm}$ anzutreffen ist. In der Jugend ist die Borke glatt. Mit zunehmendem Alter wird diese dann rauer. Die lanzettlichen Blätter sind wechselständig angeordnet und ca. $8 \mathrm{~cm}$ lang und etwa $3 \mathrm{~cm}$ breit. Junge Blätter sind nach dem Austrieb zunächst rötlichbraun, ältere Blätter sind dunkelgrün, etwas glänzend mit erhöhten Blattnerven. Die stiellosen, kleinen Blüten sitzen auf altem Holz. Unterhalb des Kelches befinden sich drei Hochblätter. Die eiförmige Frucht wird $4 \mathrm{~cm}$ lang und enthält nur einen einzigen Samen. Das wohl auffälligste Merkmal von Pycnandra acuminata ist der intensive milchig blau-grüne Latexsaft, der z. B. nach Verletzungen an der Wundstelle austritt.

Pycnandra acuminata gilt als eine der produktivsten Hyperakkumulatoren von Schwermetall, insbesondere Nickel. Ein erwachsener Baum kann (geschätzt) einen Gesamtnickelgehalt von $37 \mathrm{~kg}$ enthalten (SAGNER et al. 1998). Die Pflanze kann in verschiedenen Organen und Geweben Nickel konzentrieren. So beträgt 


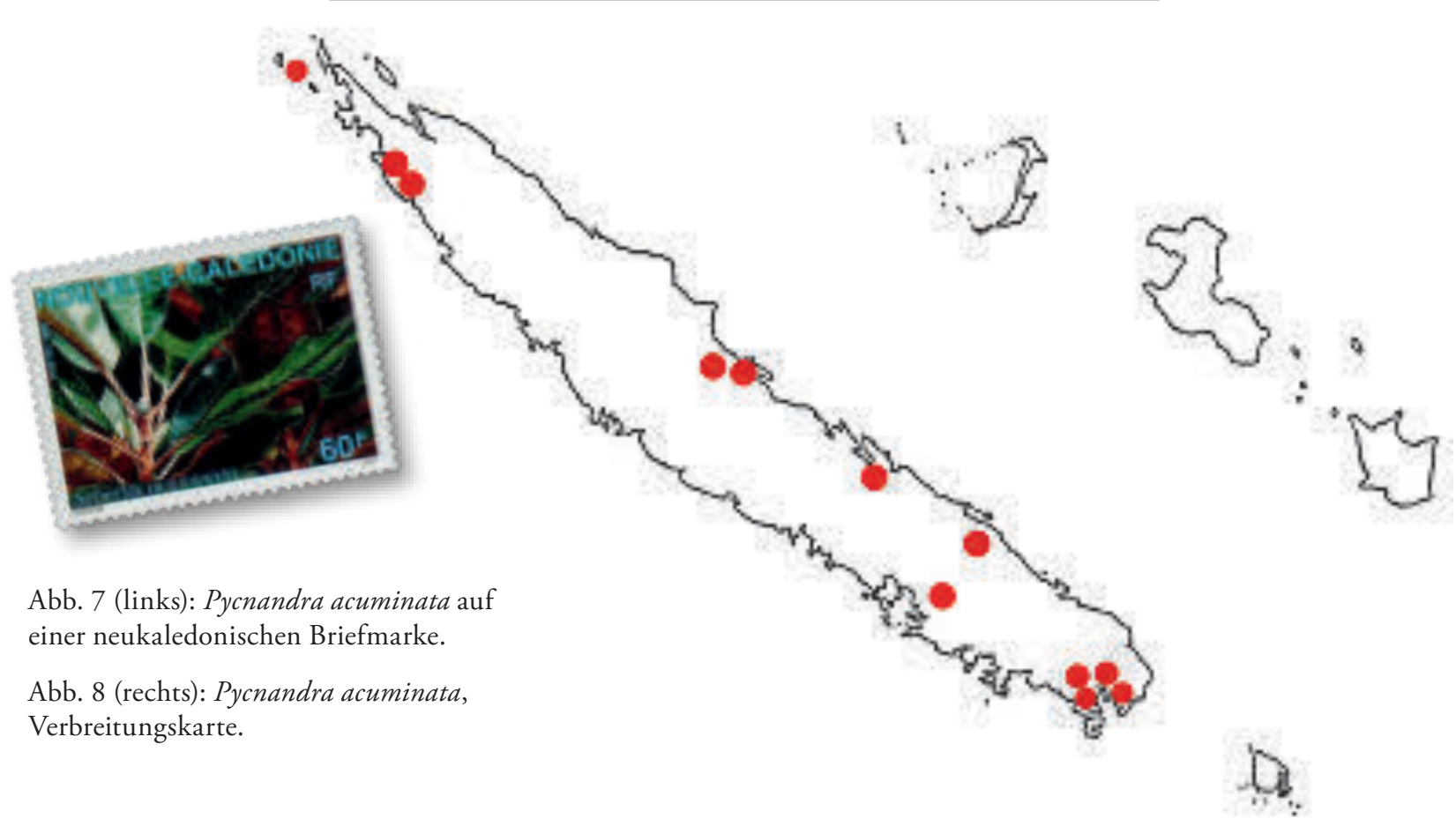

z. B. der Nickelgehalt in Blättern 1,17\% und in der Borke des Stammes sogar 2,45\% der Trockenmasse (JAffré et al. 1976). Dieser Anteil von Nickel in der Trockenmasse ist zwar mit ein bis zwei Prozent extrem hoch, aber vergleichbar mit dem der vielen weiteren neukaledonischen Hyperakkumulatoren (JAFFrÉ et al. 1976). Betrachtet man jedoch hingegen den Nickelgehalt im Latexsaft, so liegt dieser bei 11,2\% im Frischgewicht und sogar bei 25,74\% in der Trockenmasse. Citrat ist hier der Hauptligand für Nickel im Latexsaft. Damit übersteigt die Nickelkonzentration im Latexsaft den Nickelgehalt aller anderer Organe der Pflanze um ein Fünffaches (JAFFrÉ et al. 1976). Es ist daher nicht verwunderlich, dass dieser Nickelgehalt der höchste im Pflanzenreich ist und der jemals in lebendem Material nachgewiesen wurde (Jaffré et al. 1976). Pycnandra acuminata ist daher physiologisch eine interessante Art, da sie problemlos Nickelkonzentrationen ertragen kann, die für die meisten Samenpflanzen tödlich wären.

\section{Dank}

Wir danken Bernard Suprin für die großzügige Bereitstellung von Fotomaterial und die Unterstützung im Gelände auf den Forschungsreisen in Neukaledonien.

\section{Literatur}

Callahan, D. L.; Roessner, U., Dumontet, V., De Livera, A. M., Doronila, A, Baker A. J. M. \& Kolev, S. D. 2012: Elemental and metabolite profiling of nickel hyperaccumulators from New Caledonia. Phytochemistry 81: $80-89$.

Jaffré, T. Pillon, Y., Thomine, S. \& Merlot, S. 2013: The metal hyperaccumulators from New Caledonia can broad our understanding of nickel accumulation in plants. - Front Plant Sci. 4: 1-7.

Jaffré, T., Brooks, R. R., Lee, J. \& Reeves, R. D. 1976: Sebertia acuminate: A hyperaccumulator of nickel from New Caledonia. Science 193: 579-580.

Kersten W. J., Brooks, R. R., Reeves, R. D. \& Jaffré, T. 1980: Nature of nickel complexes in Psychotria douarrei and other nickel-accumulating plants. Phytochemestry.

19: $1963-1965$.

Morat, P. T., Veillon, J. M.; Rigault, F. \& Dagostini, G. 2001: Composition et caractérisation de la flore indigène de Nouvelle Calédonie (2nde édition)/Composition and characterization of the native flora of $\mathrm{New}$ Caledonia. Institut de Recherche pour le Développement. - Nouméa 2: 4.

Sagner, S., Kneer, R., Wanner, G., Cosson, J. P., Deus-Neumann, B. \& Zenk, M. H. 1998: Hyperaccumulation, complexation and distribution of nickel in Sebertia acuminata. - Phytochemistry 47: 339-347.

Schneckenburger, S. 1991: Neukaledonien, Pflanzenwelt einer Pazifikinsel. - Palmengarten Sonderheft 16

\section{Internetseiten}

Stevens, P. F. 2001 onwards: Angiosperm Phylogeny Website. Version 12, July 2012 http://www.mobot.org/ MOBOT/research/APweb/

The Plant List: Strasburgeriaceae. - in: The Plant List, Version 1.1 (September 2013) http://www.theplantlist.org/ 